\title{
Post-COVID symptoms reported at asynchronous virtual review and stratified follow-up after COVID-19 pneumonia
}

\author{
Authors: Rebecca R Taylor, ${ }^{A}$ Bhavi Trivedi, ${ }^{B}$ Neena Patel, ${ }^{C}$ Richa Singh, ${ }^{A}$ William M Ricketts, ${ }^{D}$ Katharine Elliott, ${ }^{C}$ \\ Marcus Yarwood, ${ }^{\mathrm{E}}$ Veronica White, ${ }^{\mathrm{A}}$ Hannah Hylton, ${ }^{\mathrm{F}}$ Ruth Allen, ${ }^{\mathrm{G}}$ Gavin Thomas, ${ }^{\mathrm{A}}$ Vikas Kapil, ${ }^{\mathrm{H}}$ Rachel McGuckin ${ }^{\mathrm{I}}$ \\ and Paul E Pfeffer
}

\section{Background}

The COVID-19 pandemic has strained healthcare systems and how best to address post-COVID health needs is uncertain. Here we describe the post-COVID symptoms of 675 patients followed up using a virtual review pathway, stratified by severity of acute COVID infection.

\section{Methods}

COVID-19 survivors completed an online/telephone questionnaire of symptoms after 12+ weeks and a chest $X$-ray. Dependent on findings at virtual review, patients were provided information leaflets, attended for investigations and/or were reviewed face-to-face. Outcomes were compared between patients following high-risk and low-risk admissions for COVID pneumonia, and community referrals.

Results

Patients reviewed after hospitalisation for COVID pneumonia had a median of two ongoing physical health symptoms post-COVID. The most common was fatigue $(50.3 \%$ of highrisk patients). Symptom burden did not vary significantly by severity of hospitalised COVID pneumonia but was highest in community referrals. Symptoms suggestive of depression, anxiety and post-traumatic stress disorder were common (depression occurred in $\mathbf{2 4 . 9 \%}$ of high-risk patients). Asynchronous virtual review facilitated triage of patients at highest need of face-to-face review.

Authors: ${ }^{A}$ consultant respiratory physician, Barts Health NHS Trust, London, UK; ${ }^{B}$ Clinical fellow and data analyst, Barts Health NHS Trust, London, UK; ' cspecialist training registrar in respiratory medicine, Barts Health NHS Trust, London, UK; ${ }^{\mathrm{D}}$ consultant respiratory physician, Barts Health NHS Trust, London, UK, and hon clinical senior lecturer, Barts and The London School of Medicine and Dentistry, Queen Mary University of London, UK; Edata analyst, Barts Health NHS Trust, London, UK; ' Fspecialist respiratory physiotherapist, Barts Health NHS Trust, London, UK; ${ }^{G}$ Clinical health psychologist, Barts Health NHS Trust, London, UK; ${ }^{H}$ consultant clinical pharmacologist, Barts Health NHS Trust, London, UK, and clinical senior lecturer, Barts and The London School of Medicine and Dentistry, Queen Mary University of London, UK; Istrategy lead, Barts Health NHS Trust, London, UK; J'consultant respiratory physician, Barts Health NHS Trust, London, UK, and hon senior lecturer, Barts and The London School of Medicine and Dentistry, Queen Mary University of London, UK

\section{Conclusion}

Many patients continue to have a significant burden of postCOVID symptoms irrespective of severity of initial pneumonia. How best to assess and manage long COVID will be of major importance over the next few years.

KEYWORDS: follow-up, sequalae, breathlessness, depression, long COVID

DOI: $10.7861 /$ clinmed.2021-0037

\section{Introduction}

It is established practice to review patients admitted with communityacquired pneumonia at 6-12 weeks after discharge to ascertain ongoing medical or rehabilitation needs, traditionally via face-toface consultation. ${ }^{1,2}$ During the first wave of COVID-19, caused by SARS-CoV-2, over 100,000 patients were hospitalised with COVID pneumonia in the UK and many more patients were managed in the community. A high proportion needed high-flow oxygen and many were admitted to intensive care/high dependency units. ${ }^{3}$ Experience from outbreaks of the related SARS-CoV-1 infection has identified common sequelae in survivors of breathlessness and difficulty with exertion, fatigue, difficulty with sleep, myalgia, depression and post-traumatic stress disorder (PTSD), together with functional limitations. ${ }^{4-7}$ Follow-up imaging showed interstitial lung changes, although with relatively preserved lung volumes and diffusion capacity. ${ }^{4,7}$ Early follow-up studies of severe SARS-CoV-2 pneumonia survivors have similarly shown frequent breathlessness and fatigue, and persisting interstitial radiological lung changes at 4-12 weeks post-discharge. ${ }^{8.9}$ However, by summer 2020 it was increasingly evident that many patients who had had milder acute COVID illness were nevertheless reporting significant ongoing symptoms - a syndrome now known as 'long COVID'.10

Designing follow-up pathways for these patients during a continuing pandemic has been a further challenge to stretched medical services. ${ }^{11}$ Due to the constraints of the pandemic, providing face-to-face reviews of all patients discharged after treatment for COVID-19 has not been possible in many hospitals given the large number of patients and necessity for enhanced social distancing and infection control.

To enable face-to-face review of patients with anticipated greatest clinical need, triage of discharged patients has been 
advised, although there is limited evidence to date regarding which patients are likely to have ongoing post-COVID health needs. The British Thoracic Society suggested categorisation of patients into two groups based on expectation of high or low risk of post-COVID health needs. High-risk categorisation was based on severity of acute COVID pneumonia. Admission to the intensive care unit or high dependency unit, protracted need for high concentrations of oxygen and/or ventilatory support, and/ or discharge on oxygen were deemed to be features of severe disease. Subjective clinical concerns were also to be considered. ${ }^{12,13}$

Virtual medicine can address many of these challenges, ${ }^{14,15}$ and Barts Health NHS Trust decided on a novel asynchronous virtual review pathway, designed to identify those patients with greatest need of face-to-face review while providing follow-up virtual review of all patients and personalised advice to each patient. While designed with reference to discharged inpatients, the pathway was opened up to community referrals.

Here we describe the post-COVID symptoms reported by 675 consecutive patients reviewed in our 'After COVID' virtual clinic and their follow-up assessments, stratified by severity of their acute COVID infection.

\section{Methods}

Patients

Patients admitted across Barts Health NHS Trust for presumed or confirmed COVID-19 pneumonia were electronically referred to the 'After COVID' clinic and tracked on an eDashboard (supplementary material S1). At 12 weeks after discharge, patients were invited for 'After COVID' virtual clinic review, comprising an online/telephone Amplitude questionnaire (Amplitude Clinical Outcomes, Worcestershire, UK; https://amplitude-clinical.com/) and follow-up chest X-ray. Additional referrals of patients were accepted from the community where the GP had ongoing concerns after 12 weeks following confirmed or suspected COVID in the community. Discharged patients deemed by their inpatient care team to need additional 6 -week review were telephoned by that care team at the appropriate time.

\section{Amplitude questionnaire}

The structured health screening questionnaire patients were required to complete after 12 weeks was designed with multidisciplinary and multi-specialty input from secondary care and community mental health teams. The questionnaire was digital, with patients given the option of completing online or by telephone with facilitation by trained non-clinical staff.

The Amplitude questionnaire asked patients to rate whether they currently had any of the following: shortness of breath, cough, tiredness or fatigue, problems with sleeping, memory problems, concentration difficulty, joint or muscle pain, problems with taste or smell, diarrhoea or stomach ache, or problems with appetite. For each issue, if reported as a current problem, the patient was asked to rate whether the symptom was 'getting better', 'staying the same' or 'getting worse'. For the first four issues, if reported, the patient was asked to rate severity on a 1-10 visual analogue scale (10 being very severe). The Amplitude questionnaire also included the MRC Dyspnoea Scale to assess current breathlessness on exertion and breathlessness on exertion before COVID; Generalised Anxiety Disorder scale (GAD-2) to assess anxiety symptoms; Patient Health Questionnaire (PHQ-2) for symptoms of depression; and Trauma Screening Questionnaire (TSQ) for posttraumatic stress disorder. The patients were asked whether any close family or friends had died from COVID, and their satisfaction with their quality of life. Patients were given the option to relay further concerns to the clinical team in a final free-text box.

Results were automatically imported into the electronic medical record and the 'After COVID' eDashboard was automatically updated. A list of patients who had completed the questionnaire, with or without chest X-ray, was updated on a daily basis for review by the clinical team.

\section{Asynchronous virtual clinic review}

Patients marked on the eDashboard as having completed the Amplitude questionnaire and chest $X$-ray were allocated to an available clinician to review the questionnaire answers and $X$-ray report, together with the admission details where applicable, following structured written guidance. The review was entered directly in the electronic medical record using a structured form with options boxes and a clinic letter was automatically generated to be sent to the patient with a copy to their GP.

\section{Interventions and follow-up following virtual clinic review}

All patients were sent general information leaflets on physical and mental health recovery following COVID pneumonia. In addition, dependent on the results from the questionnaire, patients were sent further information leaflets to help recovery from specific post-COVID complications (supplementary material S2) with further patient-specific information within the free text of the clinic letter. Patients were asked to attend for blood tests and/or further radiological imaging as necessary. For example, patients reporting severe fatigue were requested to have relevant blood tests. Those with appropriate ongoing rehabilitation needs were invited to enrol on the Living With COVID Recovery app digital rehabilitation programme (Living With Ltd, London, UK; www.livingwith.health/). Following the virtual clinic review patients were either discharged with advice, triaged to further online/telephone review a further 6-12 weeks later (those with moderate severity concerns), invited to early face-to-face review (high severity concerns) or referred on to other specialties. The principal criteria for further clinical review were high severity of breathlessness or reduction in functional status on reviewing the questionnaire, and/or medically concerning symptoms reported in the free-text question.

\section{Face-to-face clinic review}

Those patients triaged as needing face-to-face review were seen by a consultant respiratory physician and specialist respiratory physiotherapist. Further validated questionnaires, ECGs and blood tests were undertaken in this clinic. Breathing pattern disorder was assessed by manual assessment of respiratory motion (MARM) and the breathing pattern assessment tool (BPAT), ${ }^{16}$ and deconditioning by a one minute sit-to-stand test and clinical impression.

\section{Service evaluation}

The results reported here are from a pre-specified service evaluation of the novel pathway (Barts Health NHS Trust CEU ID 11166). Statistics were analysed in R (version 4.0.2; www.r-project.org) using 
the following additional packages: dplyr, eeptools, gplots. Clinical data were extracted from electronic medical records.

For the purposes of this evaluation, patients treated in hospital for COVID pneumonia were categorised as at 'high-risk' of postCOVID complications if they had been to intensive care during the admission or received high-flow nasal oxygen (HFNO), continuous positive airways pressure (CPAP) or non-invasive ventilation (NIV) on the medical wards. Other patients with less severe acute COVID treated in hospital for COVID pneumonia were categorised as 'low-risk'. Patients referred following community COVID pneumonia not requiring hospitalisation were categorised as 'community' referrals.

In a further analysis the patients reviewed following high-risk and low-risk admissions were combined and for each patient a symptom score of $0-10$ calculated as the number of current physical health symptoms (shortness of breath, cough, tiredness or fatigue, problems with sleeping, problems with memory, difficulty with concentration, pains in their muscles or joints, problems with sense of taste or smell, diarrhoea or stomach ache, or problems with appetite) that they reported on the Amplitude questionnaire.

\section{Results}

\section{Clinical characteristics of high-risk and low-risk patients}

675 patients had completed the Amplitude questionnaire (approximately $80 \%$ online) by the time of this evaluation (end November 2020); 169 high-risk admitted patients, 376 low-risk admitted patients and 130 community referrals (Table 1 , supplementary material S3). Both the high-risk and low-risk hospitalised groups had a male predominance, consistent with the known epidemiology of severe COVID; however, the community referral group had a significantly different female predominance (Chi-squared test; $p<0.001$ ). There was no significant difference in age between patients reviewed following high-risk and low-risk admissions (unpaired t-test, $p=0.084$ ); however, community

Table 1. Demographics of patients seen in the 'After COVID' clinic

\begin{tabular}{llll} 
& High-risk & Low-risk & Community \\
Number & 169 & 376 & 130 \\
$\begin{array}{l}\text { Gender } \\
\text { Male }\end{array}$ & $115(68 \%)$ & $222(59 \%)$ & $54(42 \%)$ \\
Age (years) & $57.1(12.9)$ & $59.3(16.2)$ & $46.8(12.9)$ \\
Ethnicity & & & \\
$\begin{array}{l}\text { White } \\
\text { Black }\end{array}$ & $55(33 \%)$ & $126(34 \%)$ & $89(68 \%)$ \\
Asian & $32(19 \%)$ & $67(18 \%)$ & $3(2 \%)$ \\
Mixed/Other & $48(28 \%)$ & $112(30 \%)$ & $14(11 \%)$ \\
Not Stated & $28(17 \%)$ & $55(15 \%)$ & $10(8 \%)$ \\
Convalescent & $16.7(6.0)$ & $16.1(6.1)$ & $23.9(6.6)$ \\
period (weeks) & & & \\
Length of & $17.0(17.4)$ & $5.9(5.9)$ & N/A \\
admission (days) & & & \\
\hline
\end{tabular}

Data presented as mean (standard deviation) or number (percentage) as appropriate referrals were significantly younger $(p<0.001)$. The hospitaladmitted patients had a broad range of ethnicities in keeping with the local East London population, whereas the community referrals had a significantly different white Caucasian predominance $(p<0.001)$

High-risk patients had had significantly longer admissions than low-risk patients (mean 17.0 vs 5.9 days; $p<0.001$ ). Patients who had been treated in hospital completed the Amplitude questionnaire an average of 16 weeks after discharge, whereas patients referred from the Community had experienced their reported acute COVID symptoms an average of 24 weeks previously $(p<0.001)$.

\section{Ongoing physical health symptoms in patients}

Patients reported symptoms across the physical health spectrum (Fig 1, Table 2). The most frequently reported symptom in all groups was fatigue, with the next most frequent in hospitalised patients being difficulty with sleep and musculoskeletal pains. In community referrals the next most frequent was breathlessness. The frequencies of physical health symptoms were similar in the high-risk and low-risk groups. However, the frequency of physical health symptoms was markedly higher in those referred from the community.

A proportion of patients reported being more breathless on exertion, as measured using the MRC Dyspnoea Scale, at post-COVID review compared to before COVID pneumonia (supplementary material S4). In $52 \%$ of patients following low-risk admissions, the MRC Dyspnoea Scores were rated at the same level before and after. A majority of patients in the high-risk group had a deterioration on the MRC Dyspnoea Scale, though 39\% of patients still rated their breathlessness at the same level on the Scale before and after. A large majority of community patients reported a worsening on the MRC Dyspnoea Scale, with only $18 \%$ rating their breathlessness at the same level before and after.

The follow-up chest $X$-ray undertaken as part of the virtual clinic review showed resolution of any admission COVID-related lung changes in $69 \%$ of high-risk patients and $83 \%$ of low-risk patients. Chest $X$-ray at review showed no abnormalities in $93 \%$ of community referrals.

\section{Ongoing mental health symptoms and quality of life}

A clinically significant minority of patients in all three groups had scores above the clinical screening thresholds on PHQ-2, GAD-2 and TSQ questionnaires, indicating that the patients had possible depression, anxiety and post-traumatic stress disorder, respectively (Table 2).

Patients were also asked to rate their satisfaction with their quality of life before and after COVID on a five-point Likert scale (supplementary material S5). More patients in both the high-risk and low-risk hospitalised groups reported they were 'satisfied' or 'very satisfied' ( $48 \%$ and $48 \%$ respectively) with their quality of life at their after COVID review than 'dissatisfied' or 'very dissatisfied' (33\% and $27 \%$ ). While the majority of community patients reported being 'satisfied' or 'very satisfied' with quality of life before COVID, more of these were 'dissatisfied' or 'very dissatisfied' (58\%), than 'satisfied' or 'very satisfied' (13\%) on review after COVID. Many patients had not returned to work following their acute COVID illness (supplementary material S6). 

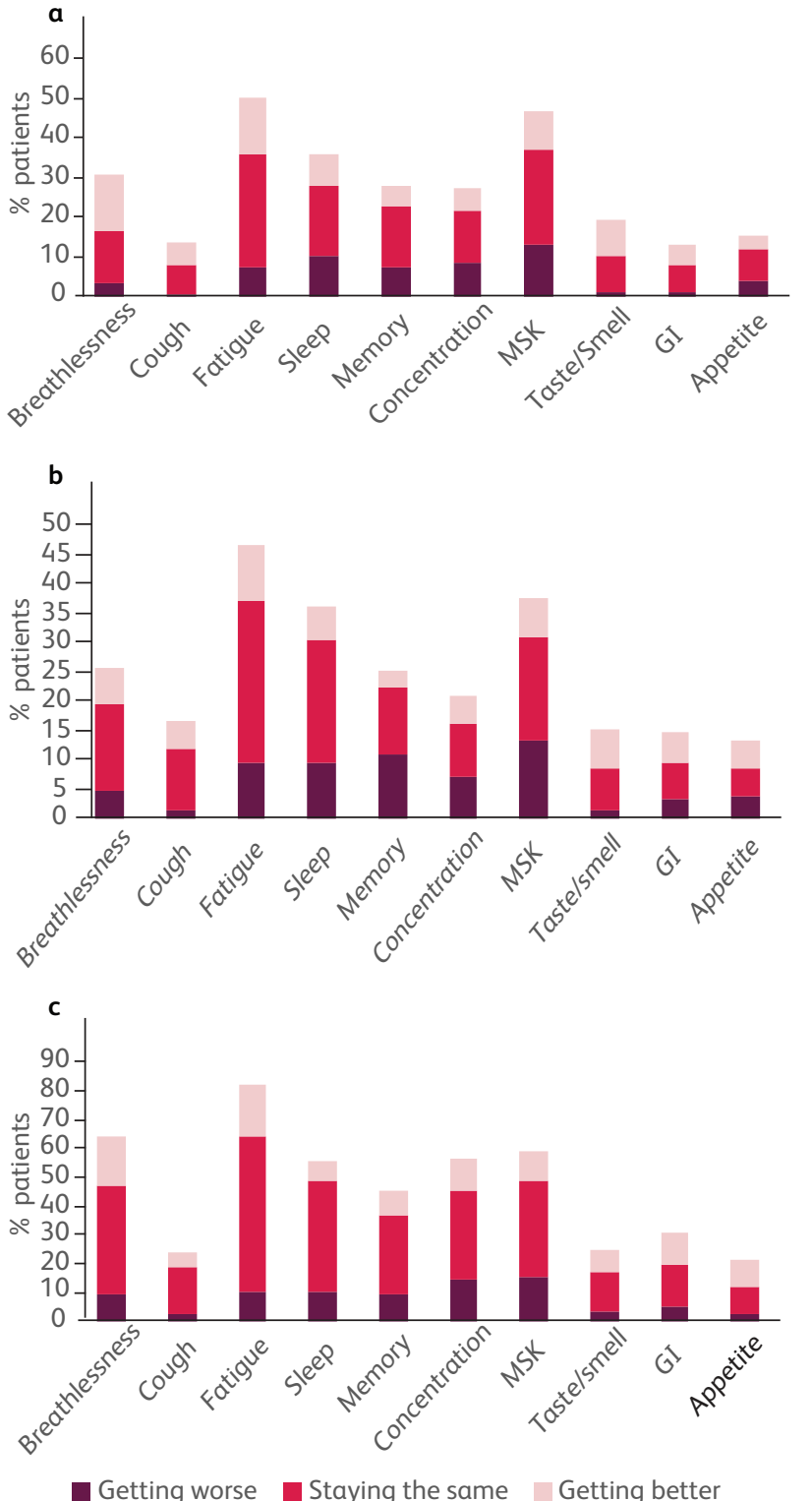

Fig 1. Physical health symptoms reported on the Amplitude questionnaire by patients at post-COVID review. (a) 'highrisk' admissions, (b) 'low-risk' admissions, (c) 'community'. Plot colours: pale pink if the symptom is 'getting better'; dark pink if the symptom is 'staying the same'; dark red if symptom is 'getting worse'. MSK = musculoskeletal muscle or joint pains; GI = gastrointestinal complaints of diarrhoea or abdominal pains.

\section{Interventions following virtual review}

Patients were sent specific advice leaflets tailored to their reported symptoms, with a similar proportion of high-risk and low-risk patients receiving each information leaflet and in general a higher proportion in the community referrals group (examples; Fig 2a). Based on raised scores on GAD-2, PHQ-2 and/or TSQ, a high proportion of patients were sent specific advice on accessing further psychological help through improving access to psychological therapies (IAPT) programmes (Fig 2b). The proportion of patients triaged after virtual clinic review for early

\section{Table 2. Physical and mental health symptoms} reported by patients at virtual review

\begin{tabular}{|c|c|c|c|}
\hline & High-risk & Low-risk & Community \\
\hline \multicolumn{4}{|l|}{ Physical health } \\
\hline Breathlessness & 30.8 & 25.5 & 64.6 \\
\hline Cough & 13.6 & 16.8 & 23.8 \\
\hline Fatigue & 50.3 & 46.8 & 82.3 \\
\hline Sleep & 36.1 & 36.2 & 55.4 \\
\hline Memory & 27.8 & 25.3 & 45.4 \\
\hline Concentration & 27.2 & 21.0 & 56.2 \\
\hline Musculoskeletal & 46.7 & 37.5 & 59.2 \\
\hline Taste/smell & 19.5 & 14.9 & 24.6 \\
\hline Gastrointestinal & 13.0 & 14.6 & 30.8 \\
\hline Appetite & 15.4 & 13.3 & 20.8 \\
\hline \multicolumn{4}{|l|}{ Mental health } \\
\hline Depression & 24.9 & 19.4 & 36.9 \\
\hline Anxiety & 20.1 & 15.2 & 33.8 \\
\hline PTSD & 16.6 & 10.9 & 26.9 \\
\hline \multicolumn{4}{|c|}{$\begin{array}{l}\text { Percentage of patients in each group reporting different physical health and } \\
\text { mental health symptoms. Physical health symptoms as directly asked in the } \\
\text { virtual questionnaire. Mental health symptoms assessed as percentage of } \\
\text { patients scoring above clinical screening threshold on PHQ-2, GAD-2 and TSQ } \\
\text { questionnaires. PTSD = post-traumatic stress disorder. }\end{array}$} \\
\hline
\end{tabular}

face-to-face clinic review or deferred telephone review was greater in the high-risk than low-risk patient groups, but highest in the community referrals (Fig 2c).

In those attending face-to-face there was a significant correlation between breathlessness scored by D12 questionnaire and by Amplitude questionnaire VAS score (Spearman's, $p=0.001$; supplementary material S7). The most common causes of breathlessness after COVID were breathing pattern disorders, deconditioning and worsening of pre-existing respiratory conditions (COPD, asthma, bronchiectasis, obesity hypoventilation syndrome, idiopathic pulmonary fibrosis). The most common causes of musculoskeletal pains were post-proning shoulder injuries and flares of pre-existing rheumatological conditions. Internal criteria for a CT chest scan were met in 67 of the 143 patients who have attended to date for face-to-face review, and of these 17 scans showed interstitial changes (supplementary material S8), although in three these were thought to pre-date COVID-19 but had possibly worsened. No new diagnoses of pulmonary emboli were made from these scans conducted 12 or more weeks after the acute illness. 53 patients were screened using the COMPASS-31 autonomic dysfunction questionnaire. ${ }^{17} 22$ patients reported moderate/severe orthostatic intolerance and 14 patients were identified as having likely autonomic dysfunction and referred onto the specialist cardiovascular autonomic clinic at Barts Heart Centre.

Factors associated with higher symptom burden post-COVID for admitted patients

In an analysis of all patients reviewed after a hospital admission, at virtual review the median number of physical health symptoms reported in the Amplitude questionnaire (symptom score) was two, 


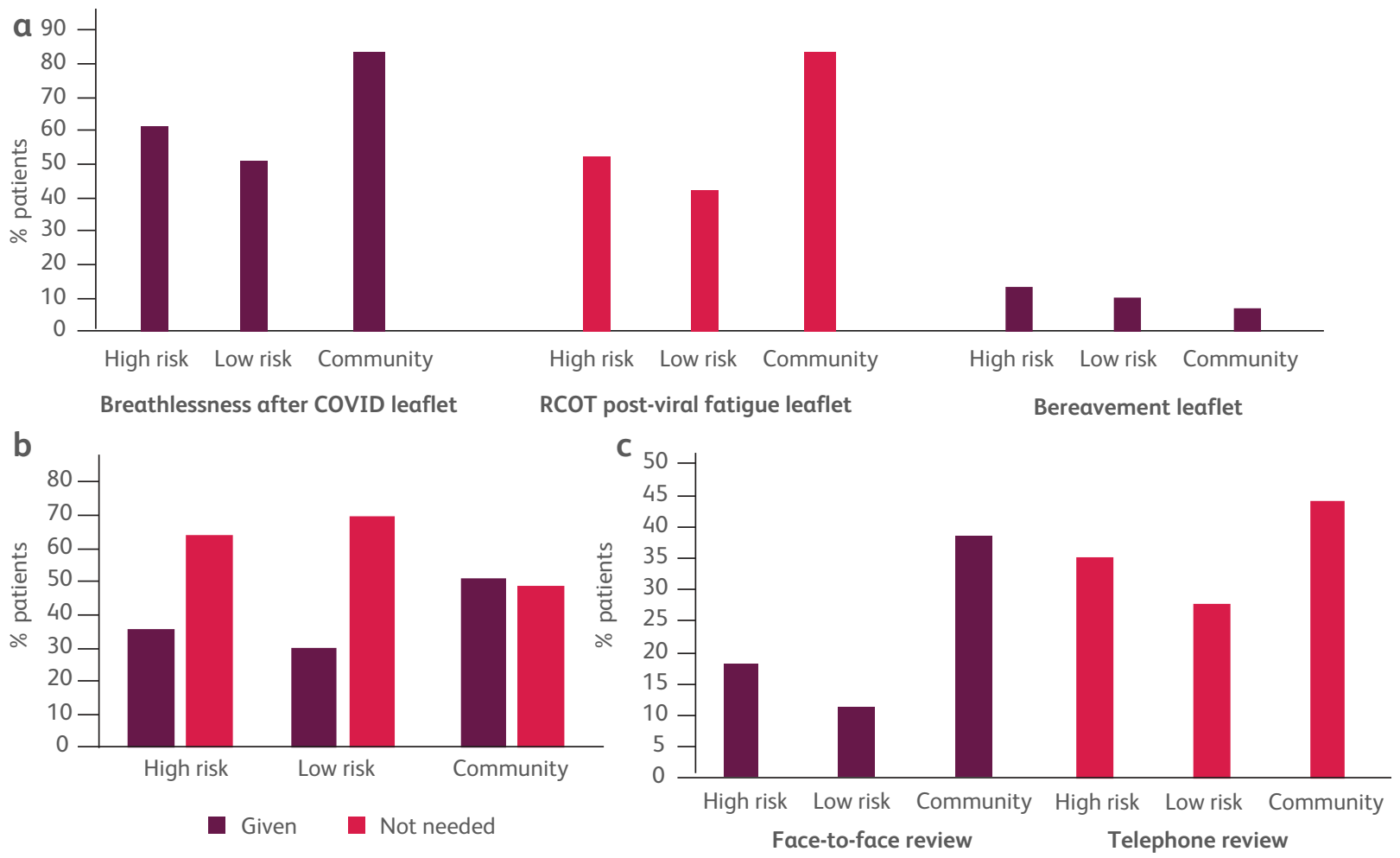

Fig 2. Clinical interventions following asynchronous Virtual Review. (a) Examples of frequency of provision of different health advice information leaflets sent to patients in different groups. Dark purple: discharged high-risk inpatients. RCOT = Royal College of Occupational Therapists. (b) All patients were sent information leaflets on mental health recovery following COVID pneumonia and a proportion more specific advice on how to access help for possible depression, anxiety or post-traumatic stress disorder based on their PHQ-2, GAD-2 and TSQ scores. (c) Proportion of patients in each group triaged to face-to-face review or telephone review after virtual clinic review of Amplitude questionnaire and chest $\mathrm{X}$-ray.

although many patients reported none (supplementary material S9, S10). There was no significant difference in symptom scores of high-risk and low-risk patients (Mann-Whitney $\cup$ Test, $p=0.29$; Fig 3a). A longer admission (7 days or more) was associated with higher symptom score than a shorter admission ( $p=0.0065$; Fig 3b), although many patients had high symptom burdens despite short admission. Female patients had significantly higher symptom scores $(p<0.001$; Fig 3c). There was no significant effect of ethnicity, age or length of convalescent period after acute COVID on symptom score (Fig 3d, supplementary material S11). Further exploratory analyses were undertaken looking for potential associations between post-COVID symptom burden and immune/inflammatory markers from blood tests during the acute admission. Although statistically significant associations were seen, for example an association between symptom score and maximum lymphocyte count during admission, correlations were not strongly predictive (supplementary material S12).

\section{Discussion}

The COVID-19 pandemic has raised major capacity issues, primarily in acute medical care but also in providing for the post-COVID health needs of thousands of patients during a pandemic. In our cohort the majority of patients continued to have one or more ongoing physical health symptoms at 12 or more weeks (average 18 weeks) after the acute infection. The frequency of different a

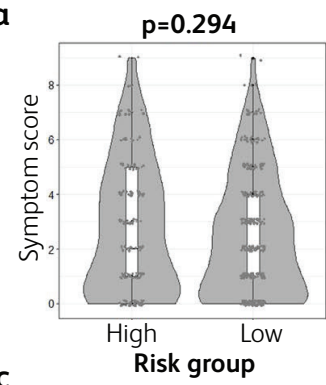

C

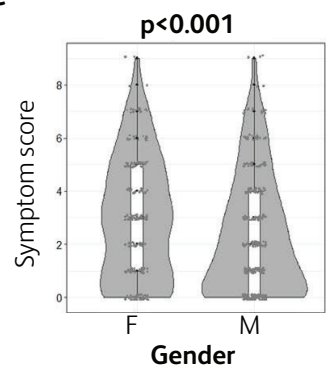

b
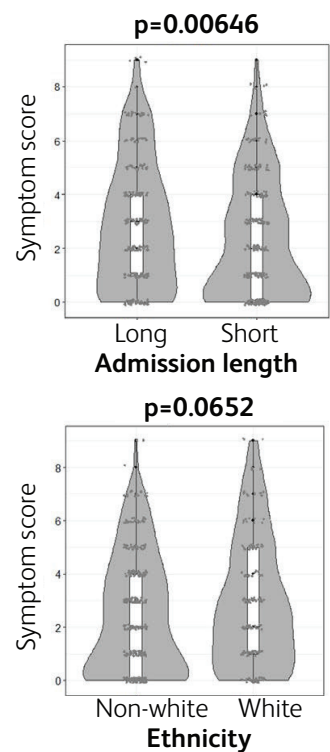

Fig 3. Factors affecting post-COVID symptom burden in patients following admission for COVID pneumonia. Violin with box-andwhisker plots of symptom scores (number of physical health symptoms reported) in patients grouped by (a) high-vs low-risk admission, (b) long ( $\geq 7$ days) vs short $(<7$ ) admissions, (c) gender and (d) ethnicity. P values for Mann-Whitney $U$ tests. 
post-COVID symptoms relative to each other was similar across all three patient groups, with fatigue the most reported symptom, as has been found in other long COVID studies. ${ }^{9,18-22}$ Some patients reported breathlessness, though on further review in many the breathlessness was an expression of fatigue, deconditioning and/ or breathing pattern disorders rather than the result of ongoing parenchymal lung pathology. Musculoskeletal pains were also prominently reported. A high prevalence of joint problems has been reported after SARS-CoV-1 infection, though many of those cases were due to osteonecrosis of the hip related to corticosteroid treatment of SARS, ${ }^{23}$ while our evaluation preceded widespread introduction of corticosteroids as treatment for COVID-19.

The prevalence of post-COVID symptoms in our patients was lower than seen in other studies that assessed patients earlier after discharge, which may reflect resolution of symptoms over time. ${ }^{8,19}$ In particular we asked our patients similar questions to Mandal and colleagues, but at a later time after discharge, and found lower frequencies for post-COVID symptoms. ${ }^{8}$ However, after 12 or more weeks many patients in our study remained highly symptomatic, with worsening symptoms in some cases. Similarly, Huang and colleagues found many patients to still have significant post-COVID symptoms at six months. ${ }^{22}$ This suggests these residual post-COVID symptoms may not resolve in some individuals without intervention.

In contrast to expectations, the health needs of patients following high-risk and low-risk hospital admissions were similar and focusing only on patients who had required ventilatory support would have missed addressing the health needs of many patients who had 'milder' acute infections but now more severe post-COVID ill health. This is consistent with other reports of high prevalence of ongoing postCOVID symptoms weeks after hospitalisation with COVID pneumonia, whether or not the patient required ventilatory support.19,21,24

Difficulty accessing post-COVID health services has been a major issue for patients, compounding their distress. ${ }^{25}$ Those patients referred from the community for COVID follow up are likely just the 'tip of the iceberg' with their symptom severity leading to a selection bias to referral, in addition to the requirement itself of ongoing symptoms/concerns for referral for community patients to this service. This likely explains the higher frequency of symptoms in community referrals. The COVID Symptom Study found long COVID to be more frequent in those who had required acute hospital attendance but nevertheless to be evident in many who never required acute hospital attendance. ${ }^{18}$

The differences in patient age, gender and ethnicity between community referrals and those reviewed following hospital admission were striking. There was an association between female gender and higher post-COVID symptom burden in our patients following a COVID admission, as has been reported in other studies. ${ }^{18,19}$ The pattern of symptoms experienced during the acute infection may also be predictive of long COVID, with, for example, the COVID Symptom Study finding headache within the first week of symptoms to be associated with increased risk of developing long COVID. ${ }^{18}$ Presence/absence of abnormalities on follow-up chest $X$-ray is not a good predictor of post-COVID health needs. ${ }^{9}$ However, the disparity in ethnicity suggests that potential inequality of access of referral from the community to long COVID clinics is an important consideration for future service design.

While some of the predictors of post-COVID symptoms assessed were statistically significant, none were strong enough to be clinically useful in predicting the post-COVID health needs of patients. This is perhaps not surprising given that long COVID is likely comprised of multiple different pathologies, with different aetiologies and different associations. ${ }^{25}$ Future larger studies may be able to distinguish different post-COVID pathologies and individual risk factors for each.

Use of an asynchronous virtual clinic review proved effective in addressing the holistic health needs of our patients following admission for COVID pneumonia, while triaging for face-to-face review a limited number of patients with more concerning postCOVID symptoms. All patients received basic information on recovery after COVID, with more specific information tailored to their individual health needs after virtual review. Selective tailored self-management information also helps overcome the dangers of information overload of patients, ${ }^{26}$ and personalisation of health advice has been shown to be beneficial in many settings. ${ }^{27,28}$ Importantly, the number of patients seen face-to-face could be accommodated within the limited resource for face-to-face reviews during the pandemic. An additional benefit for both patients and clinicians of the asynchronous virtual clinic review model is flexibility. ${ }^{15}$ Patients could complete the questionnaire when convenient for them and many clinicians reviewed patients during periods of COVID-contact self-isolation in a flexible manner.

A limitation of this service evaluation is that it relied on structured data extracted from the clinical record. The internal guideline for the virtual review was designed as a 'living guideline' with weekly review based on local and national experience, and underwent seven revisions over the 6-month period. Some information sheets and the Living With Covid Recovery App only became available in later revisions of the guideline and were not captured as structured data in all patients. We therefore cannot analyse their provision across the patient cohort. Many patients reported in the final free-text box of the questionnaire symptoms of chest discomfort, palpitations and headaches - although such symptoms were addressed in clinical review of these patients we cannot analyse their frequency in the same manner in this evaluation.

The pandemic has been a distressing time for everyone, and many people who have not had COVID have developed mental and physical health symptoms in response to the strain of living through social isolation and health fears. ${ }^{29}$ How the symptom burden in post-COVID patients may compare to the general population living through the pandemic is an interesting question that will be addressed in cohort studies such as COVIDENCE-UK. ${ }^{30}$

\section{Conclusion}

There is a high prevalence of physical health and mental health symptoms in hospital-treated patients following both 'high-risk' and 'low-risk' acute COVID pneumonia and in community-referred patients. Severity of acute pneumonia is not a good predictor of long COVID symptoms. With the current, larger, second COVID-19 wave in the UK, and increasingly stretched resources, novel pathways such as those described here will be important in the recovery of individual patients and the health service itself.

\section{Supplementary material}

Additional supplementary material may be found in the online version of this article at www.rcpjournals.org/clinmedicine: S1 - Screenshot of 'After COVID' eDashboard showing numbers of patients at different stages in the patient pathway, automatically updated each day from the electronic medical records. 
S2 - Patient information (self-management) leaflets provided to patients following virtual review.

S3 - Characteristics of patients responding to invitation to 'After COVID' review.

S4 - Reported MRC dyspnoea scores for breathlessness on exertion before (grey) and after (black) COVID pneumonia. S5 - Patient-reported satisfaction with quality of life before (grey) and after (black) COVID pneumonia.

S6-Employment (return to work) status at post-COVID assessment. S7 - Sunflower plot of D12 breathlessness scores versus Amplitude Questionnaire breathlessness severity VAS scores for those patients attending face-to-face review.

S8 - CT Chest/CT-PA findings at post-COVID Review.

S9 - Frequency histogram for number of physical health symptoms reported by patients at post-COVID review following admission for COVID pneumonia.

S10 - Heatmap of reported physical health symptoms in patients at post-COVID review following admission for COVID pneumonia. S11 - Further factors affecting post-COVID symptom burden in patients following admission for COVID pneumonia S12 - Effect of blood inflammatory and immune biomarkers during acute admission on post-COVID symptom burden in patients following admission for COVID pneumonia.

\section{Summary}

\section{What is known?}

Survivors of SARS-CoV-1 pneumonia have high rates of breathlessness, fatigue, depression, post-traumatic stress disorder (PTSD), and other health needs in the year after the acute infection.

\section{What is the question?}

$>$ Following COVID-19 pneumonia caused by SARS-CoV-2 infection, what are the ongoing health needs of survivors?

> What physical and mental health symptoms do these patients have at 12 or more weeks following the acute infection?

$>$ Do these symptoms and health needs depend on the initial severity of the acute COVID pneumonia?

$>$ Can these patients be assessed and triaged by virtual review?

\section{What was found?}

> 675 post-COVID patients were reviewed in a virtual clinic and had a median of two ongoing physical health symptoms post-COVID, the most common of which was fatigue. Musculoskeletal pains and difficulties with sleep and memory were also frequently reported.

> Depression, anxiety and post-traumatic stress disorder (PTSD) were common.

> There was no association for admitted patients between severity of acute pneumonia and post-COVID symptoms.

> Patients referred from the community had higher symptom burdens though this may reflect a selection bias in who was referred.

\section{What is the implication for practice now?}

Severity of acute admission with COVID-19 pneumonia does not help predict the post-COVID health needs of survivors but these can be assessed by virtual clinic pathways.

\section{References}

1 British Thoracic Society Standards of Care Committee. BTS guide lines for the management of community acquired pneumonia in adults. Thorax 2001;56:iv1-iv64.
2 Lim WS, Baudouin SV, George RC et al. BTS guidelines for the management of community acquired pneumonia in adults: update 2009. Thorax 2009;64:iii1-iii55.

3 Docherty AB, Harrison EM, Green CA et al. Features of 20133 UK patients in hospital with COVID-19 using the ISARIC WHO Clinical Characterisation Protocol: prospective observational cohort study. BMJ 2020;369:m1985.

4 Chan KS, Zheng JP, Mok YW et al. SARS: prognosis, outcome and sequelae. Respirology 2003;8:S36-40.

5 Moldofsky H, Patcai J. Chronic widespread musculoskeletal pain, fatigue, depression and disordered sleep in chronic post-SARS syndrome; a case-controlled study. BMC Neurol 2011;11:37.

6 Mak IW, Chu CM, Pan PC, Yiu MG, Chan VL. Long-term psychiatric morbidities among SARS survivors. Gen Hosp Psychiatry 2009;31:318-26.

7 Herridge MS, Cheung AM, Tansey CM et al. One-year outcomes in survivors of the acute respiratory distress syndrome. N Engl ] Med 2003;348:683-93.

8 Mandal S, Barnett J, Brill SE et al. 'Long-COVID': a cross-sectional study of persisting symptoms, biomarker and imaging abnormalities following hospitalisation for COVID-19. Thorax 2021;76:396-8.

9 D'Cruz RF, Waller MD, Perrin F et al. Chest radiography is a poor predictor of respiratory symptoms and functional impairment in survivors of severe COVID-19 pneumonia. ERJ Open Research 2020:00655-2020.

10 Mahase E. Covid-19: What do we know about 'long covid'? BMJ 2020;370:m2815.

11 D'Cruz RF, Perrin F, Birring SS et al. Provision of holistic care after severe COVID-19 pneumonia: anticipating clinical need and managing resources. Lancet Respir Med 2020;8:1175-6.

12 George PM, Barratt S, Desai SR et al. British Thoracic Society guidance on respiratory follow up of patients with a clinicoradiological diagnosis of COVID-19 pneumonia (Version 1.2). BTS, 2020. www.brit-thoracic.org.uk/document-library/qualityimprovement/covid-19/resp-follow-up-guidance-post-covidpneumonia/ [Accessed 31 Dec 2020].

13 George PM, Barratt SL, Condliffe R et al. Respiratory follow-up of patients with COVID-19 pneumonia. Thorax 2020;75:1009-16.

14 Hare N, Bansal P, Bajowala SS et al. Work Group Report: COVID-19: Unmasking telemedicine. J Allergy Clin Immunol Pract 2020;8:2461-73.

15 Duffy S, Lee TH. In-person health care as option B. N Engl J Med 2018;378:104-6.

16 Todd S, Walsted ES, Grillo L et al. Novel assessment tool to detect breathing pattern disorder in patients with refractory asthma. Respirology 2018;23:284-90.

17 Sletten DM, Suarez GA, Low PA, Mandrekar ], Singer W. COMPASS 31: a refined and abbreviated Composite Autonomic Symptom Score. Mayo Clin Proc 2012;87:1196-201.

18 Sudre CH, Murray B, Varsavsky T et al. Attributes and predictors of Long-COVID. Nat Med 2021;27:626-31.

19 Halpin SJ, McIvor C, Whyatt G et al. Postdischarge symptoms and rehabilitation needs in survivors of COVID-19 infection: a crosssectional evaluation. J Med Virol 2021;93:1013-22.

20 Carfi A, Bernabei R, Landi F, Group GAC-P-ACS. Persistent symptoms in patients after acute COVID-19. JAMA 2020;324:603-5.

21 Townsend L, Dyer AH, Jones $\mathrm{K}$ et al. Persistent fatigue following SARS-CoV-2 infection is common and independent of severity of initial infection. PLoS One 2020;15:e240784.

22 Huang C, Huang L, Wang Y et al. 6-month consequences of COVID-19 in patients discharged from hospital: a cohort study. Lancet 2021;397:220-32.

23 Griffith JF. Musculoskeletal complications of severe acute respiratory syndrome. Semin Musculoskelet Radiol 2011;15:554-60.

24 Arnold DT, Hamilton FW, Milne A et al. Patient outcomes after hospitalisation with COVID-19 and implications for follow-up: results from a prospective UK cohort. Thorax 2021;76:399-401. 
25 Ladds E, Rushforth A, Wieringa S et al. Persistent symptoms after Covid-19: qualitative study of 114 'long Covid' patients and draft quality principles for services. BMC Health Serv Res 2020;20:1144.

26 Liu CF, Kuo KM. Does information overload prevent chronic patients from reading self-management educational materials? Int J Med Inform 2016:89:1-8.

27 de Nooijer J, Lechner L, Candel M, de Vries H. Short- and long-term effects of tailored information versus general information on determinants and intentions related to early detection of cancer. Prev Med 2004;38:694-703.

28 Sutton S, Gilbert H. Effectiveness of individually tailored smoking cessation advice letters as an adjunct to telephone counselling and generic self-help materials: randomized controlled trial. Addiction 2007:102:994-1000
29 Pierce M, Hope H, Ford T et al. Mental health before and during the COVID-19 pandemic: a longitudinal probability sample survey of the UK population. Lancet Psychiatry 2020;7:883-92.

30 COVIDENCE UK Collaboration. About the COVIDENCE UK Study. www.qmul.ac.uk/covidence/about-the-covidence-uk-study/ [Accessed 11 March 2021].

Address for correspondence: Dr Paul Pfeffer, consultant respiratory physician, Department of Respiratory Medicine, St Bartholomew's Hospital, West Smithfield, London EC1A 7BE, UK.

Email: p.pfeffer@qmul.ac.uk Twitter: @DoctorPfeff 